\title{
A note on once reinforced random walk on ladder $\mathbb{Z} \times\{0,1\}^{*}$
}

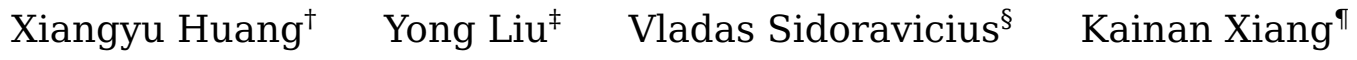

\begin{abstract}
Given any $\delta \in(0, \infty)$, let $\left(X_{n}\right)_{n=0}^{\infty}$ be the $\delta$-once reinforced random walk on ladder $\mathbb{Z} \times\{0,1\}$ with the following edge weight function at the $(n+1)$-th step:

$$
w_{n}(e)=1+(\delta-1) \cdot I_{\{N(e, n)>0\}}= \begin{cases}1 & \text { if } N(e, n)=0 \\ \delta & \text { if } N(e, n)>0 .\end{cases}
$$

Here $N(e, n):=\#\left\{i<n: X_{i} X_{i+1}=e\right\}$ is the number of times that edge $e$ has been traversed by the walk before time $n$. It was proved that $\left(X_{n}\right)_{n=0}^{\infty}$ is almost surely recurrent for $\delta>1 / 2$ (Vervoort (2002) [8] and Sellke (2006) [7]), while the a.s. recurrence for negative reinforcement factor $\delta \in(0,1 / 2]$ remained open. In this note, we give an affirmative answer to this question.
\end{abstract}

Keywords: once reinforced random walk; recurrence; ladder. MSC2020 subject classifications: $60 \mathrm{~K} 35$.

Submitted to ECP on October 9, 2020, final version accepted on June 2, 2021.

\section{Introduction and main result}

Let $\mathbb{Z}$ (resp. $\mathbb{N}$ ) be the set of all integers (resp. natural numbers). For any $n \in \mathbb{N}$, let $\mathbb{Z} \times\{0, \ldots, n\}$ be a ladder graph with $n+1$ levels, where two vertices $\left(x_{1}, y_{1}\right)$ and $\left(x_{2}, y_{2}\right)$ are adjacent if and only if $\left|x_{1}-x_{2}\right|+\left|y_{1}-y_{2}\right|=1$. In this note, we devote to investigate recurrence of once negatively reinforced random walk on ladder $\mathbb{Z} \times\{0,1\}$.

To begin, let $G=(V, E)$ be a connected locally finite graph with vertex set $V$ and edge set $E$. When two vertices $u$ and $v$ are adjacent, denoted by $u \sim v$, we denote by $u v$ the undirected edge connecting $u$ and $v, \overrightarrow{u v}$ the directed edge from $u$ to $v$. The edge reinforced random walk (ERRW) on $G$ is a stochastic process $\mathbf{X}=\left(X_{n}\right)_{n=0}^{\infty}$ in $V$ with the following transition probability:

$$
\mathbb{P}\left(X_{n+1}=u \mid \mathscr{F}_{n}\right)=\left\{\begin{array}{cc}
\frac{w_{n}(u v)}{\sum_{u^{\prime} \sim v} w_{n}\left(u^{\prime} v\right)}, & \text { on }\left\{X_{n}=v\right\}, u \sim v, \\
0, & \text { otherwise },
\end{array}\right.
$$

${ }^{*}$ Y. Liu is supported by CNNSF (No. 11731009, No.11926327) and Center for Statistical Science, PKU. K. Xiang is supported by CNNSF (No. 11671216) and by Hu Xiang Gao Ceng Ci Ren Cai Ju Jiao Gong Cheng-Chuang Xin Ren Cai (No. 2019RS1057).

${ }^{\dagger}$ LMAM, School of Mathematical Sciences, Peking University, Beijing 100871, China. E-mail: hxy19930702@ pku. edu.cn

${ }^{\ddagger}$ LMAM, School of Mathematical Sciences, Peking University, Beijing 100871, China. E-mail: liuyong@math . pku. edu.cn

${ }^{\S}$ Courant Institute of Mathematical Sciences, New York, NY 10012, USA; NYU-ECNU Institute of Mathematical Sciences, NYU-Shanghai, China. E-mail: vs1138@nyu.edu

"School of Mathematics and Computational Science, Xiangtan University, Xiangtan City 411105, Hunan Province, China. E-mail: kainan.xiang@xtu.edu.cn 
where $\left(\mathscr{F}_{n}\right)_{n \geq 0}$ is the natural filtration generated by the history of $\mathbf{X}$, i.e. $\mathscr{F}_{n}=$ $\sigma\left(X_{0}, \ldots, X_{n}\right)$ for any integer $n \geq 0$, and $w_{n}(u v)$ is an $\mathscr{F}_{n}$-measurable random weight of edge $u v$ at the $(n+1)$-th step.

The original model of reinforced random walk in literature was firstly introduced by Coppersmith and Diaconis in 1987 [1]. They considered the following weight function:

$$
w_{n}(e)=1+N(e, n) \cdot \delta
$$

where $\delta>0$ is the reinforcement factor and $N(e, n)=\#\left\{i<n: X_{i} X_{i+1}=e\right\}$ is the number of times that edge $e$ has been traversed before time $n$.

Davis (1990) [3] introduced the $\delta$-once reinforced random walk (ORRW), which is an interesting variant of ERRW with weight

$$
w_{n}(e)=1+(\delta-1) \cdot I_{\{N(e, n)>0\}}= \begin{cases}1 & \text { if } N(e, n)=0 \\ \delta & \text { if } N(e, n)>0\end{cases}
$$

It takes value $\delta$ if and only if the edge has been crossed, and 1 otherwise. We call ORRW positively reinforced if $\delta>1$ and negatively reinforced if $\delta \in(0,1)$. While it seems to be a simpler model than the other ERRWs since the weight function is simple, ORRW has less results and no general methods are developed for its study. We consider transience/recurrence of this stochastic process. A sample realization of a random walk is said to be transient (resp. recurrent) if every vertex is visited only finitely many times (resp. infinitely often) (see [8, Definition 2]). ORRW is recurrent a.s. on $\mathbb{Z}^{1}$ for any $\delta>0$. However, no transience/recurrence result of ORRW is currently known on $\mathbb{Z}^{d}$ with $d \geq 2$. Kious and Sidoravicius (2018) [6] showed a transience/recurrence phase transition for ORRW on $\mathbb{Z}^{d}$-like trees. To our knowledge, it is the first example of phase transition for ORRW. Then Collevecchio, Kious and Sidoravicius (2020) [2] proved a very elegant result: ORRW on general trees $\mathcal{T}$ has a transience/recurrence phase transition. The critical point is exactly the following branching-ruin number:

$$
\operatorname{br}_{r}(\mathcal{T})=\sup \left\{\lambda>0: \inf _{\pi \in \Pi} \sum_{e \in \pi}|e|^{-\lambda}>0\right\},
$$

where $\Pi$ is the set of cutsets separating the root from infinity, and $|e|$ is the distance between $e$ and the root. It completes the whole work for transience/recurrence of the ORRW on trees.

When considering ORRW on $\mathbb{Z}^{d}$, Sidoravicius conjectured that it is recurrent with $d=2$ and undergoes a phase transition for any $d \geq 3$. However, it is still an open problem and there is no result on transience/recurrence for any $\delta>0$. Since $\mathbb{Z}^{2}$ is the asymptotic graph of $\mathbb{Z} \times\{-n, \ldots,-1,0,1, \ldots, n\}$ as $n \rightarrow \infty$, it leads to the study of ORRW on general ladders $\mathbb{Z} \times \Gamma$ with $\Gamma$ being a finite connected graph. To the best of our knowledge, the complete depiction on transience/recurrence under this setting remains an open problem, even for the simplest case $\mathbb{Z} \times\{0,1\}$.

In 1994, Sellke [7] showed that the ORRW on $\mathbb{Z} \times\{0,1, \ldots, n\}$ is recurrent almost surely for any $\delta \in(1, n /(n-1))$; and in particular it is recurrent almost surely for any $\delta \in(1, \infty)$ when $n=1$. Afterwards Vervoort (2002) [8] verified the recurrence of ORRW for $\delta \in(n /(n+1), 1)$, and then claimed that there exist $\delta_{1}, \delta_{2}>0$ with $\delta_{1}<1-1 /(n+1)<$ $1+1 /(n-1)<\delta_{2}$ such that the ORRW is a.s. recurrent for any $\delta \in\left(\delta_{1}, \delta_{2}\right)$. These results offered partial characterizations on the phase space when reinforcement parameters are small. Intuitively, when the reinforcement factor $\delta$ is large enough, the walk will have a strong tendency to cross the edge traversed before. That is to say, the ORRW prefers to stick around the origin, which naturally deduces recurrence. Kious, Schapira and Singh 
(2018) [5] proved that there exists a constant $C>0$ such that for any finite connected graph $\Gamma$, the ORRW on $\mathbb{Z} \times \Gamma$ is recurrent when $\delta \geq 1+C|\Gamma|^{40}$.

In this note, we prove the following result which completely confirms the recurrence on the simplest ladder $\mathbb{Z} \times\{0,1\}$.

Theorem 1.1. Let $\mathbf{X}=\left(X_{n}\right)_{n=0}^{\infty}$ be an ORRW on ladder $\mathbb{Z} \times\{0,1\}$ with initial site $(0,0)$ and reinforcement factor $\delta \in(0,1)$. Then $\mathbf{X}$ is recurrent almost surely.

This note is organized as follows: In Section 2, inspired by Vervoort [8, Lemma 11], we prove a criterion for recurrence of ORRWs on infinite connected locally finite graphs, see Theorem 2.1. In Section 3, based on Theorem 2.1, we prove Theorem 1.1 by a novel estimate of the ORRWs on $\mathbb{Z} \times\{0,1\}$ specified in Lemma 3.2. Section 4 is a short conclusion.

\section{Criterion for recurrence of ORRWs}

We start with some further notations. For $h$ a function defined on $V$, and for any directed edge $\overrightarrow{v u}$ of $G$, define $\Delta_{h}(\overrightarrow{v u})=h(u)-h(v)$. We say $h: V \rightarrow \mathbb{R}$ is a superharmonic (resp. harmonic) function if

$$
\sum_{u \sim v} \Delta_{h}(\overrightarrow{v u}) \leq 0(\text { resp. }=0), \forall v \in V
$$

Then for $t \in \mathbb{N}$, define random sets $E_{t}$ and $A_{t}$ (Vervoort [8, Definition 10]),

$$
\begin{aligned}
& E_{t}=\left\{X_{s} X_{s+1}: s<t\right\} \\
& A_{t}=\left\{\overrightarrow{v u}: v u \in E_{t}, \overrightarrow{v u}=\overrightarrow{X_{s} X_{s+1}} \text { for } s=\min \left\{s^{\prime}<t: X_{s^{\prime}} X_{s^{\prime}+1}=v u\right\}\right\} .
\end{aligned}
$$

That is to say, $E_{t}$ is an edge set containing the edges that have been traversed up to time $t$ and $A_{t}$ is an arc set obtained from $E_{t}$ by orienting each edge according to the direction in which it was firstly traversed.

Theorem 2.1. Let $G=(V, E)$ be an infinite connected locally finite graph and $h: V \rightarrow \mathbb{R}$ be a function satisfying that

- $h$ is superharmonic everywhere except on a finite subset $F \subset V$,

- $h(v) \rightarrow+\infty$ as $v$ goes to infinity.

Consider $\delta$-ORRW $\mathbf{X}$ on $G$ starting at a vertex $v_{0}$, and denote by $\eta_{r}:=\inf \left\{t: h\left(X_{t}\right) \geq r\right\}$ and $\tau_{r^{\prime}}:=\inf \left\{t \geq \eta_{r}: h\left(X_{t}\right) \geq r^{\prime}\right.$ or $\left.X_{t} \in F\right\}$. If for some $\varepsilon>0$, any $r>h\left(v_{0}\right)$ and any $r_{0} \in \mathbb{R}$, there exists a $r^{\prime}>r_{0}$ (i.e., there exists a sequence of real numbers $r^{\prime} \uparrow \infty$ ) such that

$$
(\delta-1) \mathbb{E}\left(\sum_{\overrightarrow{v u} \in A_{\tau_{r^{\prime}}} \backslash A_{\eta_{r}}} \Delta_{h}(\overrightarrow{v u}) \mid \mathscr{F}_{\eta_{r}}\right) \geq-(1-\varepsilon) r^{\prime}, \text { a.s. }
$$

then $\mathbf{X}$ is recurrent almost surely.

Remark 2.2. Theorem 2.1 inherits the spirit of [8, Lemma 11]. To the best of our knowledge, Vervoort's proof of [8, Lemma 11] is not completely precise and not easily to be corrected since he used the deterministic time $t_{0}$. Therefore, we cannot use this result directly and have to show Theorem 2.1 in details. In this theorem, we replace "fixed time $t_{0}$ " and " $\sum_{\overrightarrow{v u} \in A_{\tau_{r}}}$ " in (57) of [8, Lemma 11] by stopping time $\eta_{r}$ and $\sum_{\overrightarrow{v u} \in A_{\tau_{r^{\prime}}} \backslash A_{\eta_{r}}}$ respectively, and remove " $-c$ " on the right hand side (RHS) of (57) of [8, Lemma 11].

Before proving this theorem, we show some lemmas. 
Lemma 2.3. For $\delta$-ORRW $\mathbf{X}$ on finite connected graph $G_{0}=\left(V_{0}, E_{0}\right)$, denote vertex cover time by

$$
\rho_{V_{0}}:=\inf \left\{t: \forall v \in V_{0}, \exists s \leq t, X_{s}=v\right\}
$$

Then $\rho_{V_{0}}<\infty$ a.s.

We omit the proof of Lemma 2.3 since it is straightforward. Inspired by [8, Lemma 6-7], we obtain the following lemma.

Lemma 2.4. Given an infinite connected locally finite graph $G=(V, E)$. Let $\mathbf{X}$ be the $\delta$-ORRW on $G$ starting from $v_{0} \in V$. For any finite connected subgraph with vertex set $F \subset V$, the following two statements are equivalent.

(a) $F$ is visited infinitely often by $\mathbf{X}$ almost surely.

(b) $\mathrm{X}$ is recurrent almost surely.

Proof. Since (b) $\Longrightarrow(\mathbf{a})$ is straightforward, we thus concentrate on the converse direction.

Assume (a) holds. Due to $F$ being finite, there is at least one random vertex $v \in F$ which is visited infinitely often by $\mathbf{X}$ almost surely. Hence, to prove (b), it suffices to prove that

$\mathbb{P}(v$ is visited infinitely often and $u$ only finitely often by $\mathbf{X})=0, v \in F, u \in V$.

Moreover, once showing that (2.2) holds for all $u \sim v$, we may further verify the equation through (2.2) for any $v \in F$ and $u \in V$ by the connectivity of $G$ and induction on the graph distance $d_{G}(v, u)$. Thus we are to concentrate on the case of adjacent vertices.

Let $\mathcal{A}_{t_{0}, t}^{k}(u, v)$ be the event that $u$ is not visited by $\mathbf{X}$ in time interval $\left[t_{0}, t\right]$ and that $v$ is visited by $\mathbf{X}$ for $k$ times in $\left[t_{0}, t-1\right]$. For any visit time $s \in\left[t_{0}, t-1\right]$ of $\mathbf{X}$ to $v$,

$$
\sum_{v^{\prime} \sim v} w_{s}\left(v^{\prime} v\right) \leq\left(\#\left\{v^{\prime}: v^{\prime} \sim v\right\}\right) \cdot(\delta \vee 1) \text { and } w_{s}(u v) \geq \delta \wedge 1 .
$$

Thus the probability of $\mathbf{X}$ not immediately traversing $u v$ just after time $s$ is at most $1-c$, where

$$
c=\frac{\delta \wedge 1}{\left(\#\left\{v^{\prime}: v^{\prime} \sim v\right\}\right) \cdot(\delta \vee 1)} \in(0,1] .
$$

Therefore, by induction on $k$,

$$
\mathbb{P}\left(\mathcal{A}_{t_{0}, t}^{k}(u, v)\right) \leq(1-c)^{k} .
$$

If we first let $t \uparrow \infty$ and then $k \uparrow \infty$, we see that

$\mathbb{P}\left(v\right.$ is visited infinitely often and $u$ never by $\mathbf{X}$ after time $\left.t_{0}\right) \leq \lim _{k \uparrow \infty}(1-c)^{k}=0$.

Summing over all $t_{0} \geq 0$, we get the desired result.

Note that

$$
N(e, n)=\#\left\{i: X_{i} X_{i+1}=e, 0 \leq i \leq n-1\right\}
$$

is the number of times that edge $e$ has been traversed before time $n$ and $w_{n}(e)=$ $1+(\delta-1) \cdot I_{\{N(e, n)>0\}}$. For any $V^{\prime} \subset V$, define

$$
M_{t}=\sum_{s=0}^{t-1}\left\{\begin{array}{lc}
\frac{\Delta_{h}\left(\overrightarrow{X_{s} X_{s+1}}\right)}{w_{s}\left(X_{s} X_{s+1}\right)}, & X_{s} \in V^{\prime}, \\
0, & \text { otherwise. }
\end{array}\right.
$$

We recall the following two lemmas from Vervoort [8], which are not hard to verify. 
Lemma 2.5 (Vervoort [8] Lemma 9). For any infinite connected locally finite graph $G$ and $\delta$-ORRW $\mathbf{X}$ on $G$, if $h: V \rightarrow \mathbb{R}$ is superharmonic function, then $\left(M_{t}\right)_{t=0}^{\infty}$ is a supermartingale.

Proof. If $X_{t} \in V^{\prime}$ then $M_{t+1}=M_{t}$, otherwise

$$
\begin{aligned}
M_{t} & \geq M_{t}+\frac{1}{\sum_{u \sim X_{t}} w_{t}\left(X_{t} u\right)} \sum_{u \sim X_{t}} \Delta_{h}\left(\overrightarrow{X_{t} u}\right) \\
& =M_{t}+\sum_{v \sim X_{t}} \frac{w_{t}\left(X_{t} v\right)}{\sum_{u \sim X_{t}} w_{t}\left(X_{t} u\right)} \frac{\Delta_{h}\left(\overrightarrow{X_{t} v}\right)}{w_{t}\left(X_{t} v\right)} \\
& =M_{t}+\sum_{v \sim X_{t}} \mathbb{P}\left(X_{t+1}=v \mid \mathscr{F}_{t}\right) \frac{\Delta_{h}\left(\overrightarrow{X_{t} v}\right)}{w_{t}\left(X_{t} v\right)} \\
& =\mathbb{E}\left(M_{t+1} \mid \mathscr{F}_{t}\right) .
\end{aligned}
$$

Lemma 2.6 (Vervoort [8] Lemma 10). Given an infinite connected locally finite graph $G$ and the $\delta$-ORRW $\mathbf{X}$ on $G$. Let $M_{t}$ be given by (2.3) for some $h: V \rightarrow \mathbb{R}$. Then for any $t \geq s$,

$$
\delta\left(M_{t}-M_{s}\right)=h\left(X_{t}\right)-h\left(X_{s}\right)+(\delta-1) \sum_{\overrightarrow{v u} \in A_{t} \backslash A_{s}} \Delta_{h}(\overrightarrow{v u})
$$

on the event that the set $V \backslash V^{\prime}$ has not been visited in the interval $[s, t)$.

Proof. Since $V \backslash V^{\prime}$ has not been visited at any time $t^{\prime}$ with $s \leq t^{\prime}<t, M_{t}-M_{s}=$ $\sum_{t^{\prime}=s}^{t-1} \frac{\Delta_{h}\left(\overrightarrow{X_{t^{\prime}} X_{t^{\prime}+1}}\right)}{w_{t^{\prime}}\left(X_{t^{\prime}} X_{t^{\prime}+1}\right)}$. Note that for $t^{\prime} \in[s, t), w_{t^{\prime}}\left(X_{t^{\prime}} X_{t^{\prime}+1}\right)=1$ if and only if $\overrightarrow{X_{t^{\prime}} X_{t^{\prime}+1}} \in$ $A_{t} \backslash A_{s}$. Moreover, for every $\overrightarrow{v u} \in A_{t} \backslash A_{s}$ there exists a unique $t^{\prime} \in[s, t)$ such that $\overrightarrow{X_{t^{\prime}} X_{t^{\prime}+1}}=\overrightarrow{v u}$, which establishes a map $\overrightarrow{v u} \mapsto t^{\prime}$. In addition, it is a bijection from $A_{t} \backslash A_{s}$ to $\left\{t^{\prime} \in[s, t): \overrightarrow{X_{t^{\prime}} X_{t^{\prime}+1}} \in A_{t} \backslash A_{s}\right\}$. Hence

$$
\begin{aligned}
& M_{t}-M_{s}=\sum_{\substack{s \leq t^{\prime}<t, \overrightarrow{X_{t^{\prime}} X_{t^{\prime}+1}^{\longrightarrow}} \notin A_{t} \backslash A_{s}}} \frac{\Delta_{h}\left(\overrightarrow{X_{t^{\prime}} X_{t^{\prime}+1}}\right)}{\delta}+\sum_{\substack { s \leq t^{\prime}<t,{c}{X_{t^{\prime} X_{t^{\prime}+1}}^{\longrightarrow} \in A_{t} \backslash A_{s}{ s \leq t ^ { \prime } < t , \\
\begin{subarray} { c } { X _ { t ^ { \prime } X _ { t ^ { \prime } + 1 } } ^ { \longrightarrow } \in A _ { t } \backslash A _ { s } } }\end{subarray}} \Delta_{h}\left(\overrightarrow{X_{t^{\prime}} X_{t^{\prime}+1}}\right) \\
& =\sum_{t^{\prime}=s}^{t-1} \frac{\Delta_{h}\left(\overrightarrow{X_{t^{\prime}} X_{t^{\prime}+1}}\right)}{\delta}+\left(1-\frac{1}{\delta}\right) \sum_{\substack { s \leq t^{\prime}<t,{c}{X_{t^{\prime} X_{t^{\prime}+1}} \in A_{t} \backslash A_{s}{ s \leq t ^ { \prime } < t , \\
\begin{subarray} { c } { X _ { t ^ { \prime } X _ { t ^ { \prime } + 1 } } \in A _ { t } \backslash A _ { s } } }\end{subarray}} \Delta_{h}\left(\overrightarrow{X_{t^{\prime}} X_{t^{\prime}+1}}\right) \\
& =\sum_{t^{\prime}=s}^{t-1} \frac{\Delta_{h}\left(\overrightarrow{X_{t^{\prime}} X_{t^{\prime}+1}}\right)}{\delta}+\left(1-\frac{1}{\delta}\right) \sum_{\overrightarrow{v u} \in A_{t} \backslash A_{s}} \Delta_{h}(\overrightarrow{v u}) \\
& =\frac{h\left(X_{t}\right)-h\left(X_{s}\right)}{\delta}+\left(1-\frac{1}{\delta}\right) \sum_{\overrightarrow{v u} \in A_{t} \backslash A_{s}} \Delta_{h}(\overrightarrow{v u}),
\end{aligned}
$$

which implies the result.

Proof of Theorem 2.1. By Lemma 2.4, we only need to show $F$ is visited infinitely often by $\mathbf{X}$ almost surely. To this end, it is enough to show that there is constant $c>0$ such that for all $r>0$, there exists a $\hat{r}>r$ with

$$
\mathbb{P}\left(X_{\tau_{\hat{r}}} \in F \mid \mathscr{F}_{\eta_{r}}\right) \geq c \text { a.s. }
$$

In fact, choosing a sequence of $r_{n} \uparrow \infty$ such that $\mathbb{P}\left(X_{\tau_{r_{n+1}}} \in F \mid \mathscr{F}_{\eta_{r_{n}}}\right) \geq c$ a.s. successively, we can obtain the theorem by the conditional Borel-Cantelli lemma ([4] Theorem 5.3.2) since $\eta_{r_{n}} \rightarrow \infty$ as $n \rightarrow \infty$. 
Now, we determine $c$ and $\hat{r}$ satisfying (2.5) by (2.1). Without loss of generality, assume $h \geq 0$. Noting that $G=(V, E)$ is locally finite and $h(v) \rightarrow+\infty$ as $v$ goes to infinity, by condition of Theorem 2.1, for any $r>h\left(v_{0}\right)$, we can find a deterministic $r^{\prime}>\frac{2 h\left(X_{\eta_{r}}\right)}{\varepsilon} \vee(r+1)$ such that (2.1) holds.

Set ${ }^{\varepsilon} M_{t}$ to be the supermartingale in (2.3) with $V^{\prime}:=V \backslash F$. Due to Lemma 2.3, it is clear that $\eta_{r}, \tau_{r^{\prime}}<\infty$ almost surely. Since $A_{\eta_{r}} \subset\{\overrightarrow{v u}: h(v)<r\}$ and

$$
A_{t} \backslash A_{\eta_{r}} \subset\left\{\overrightarrow{v u}: v \notin F, h(v)<r^{\prime}\right\} \text { for any } \eta_{r} \leq t \leq \tau_{r^{\prime}},
$$

Lemma 2.6 implies that $M_{t}$ is bounded for any $\eta_{r} \leq t \leq \tau_{r^{\prime}}$. Thus by Lemma 2.5 and the optional stopping time theorem we know that $\mathbb{E}\left(\delta M_{\tau_{r^{\prime}}} \mid \mathscr{F}_{\eta_{r}}\right) \leq \delta M_{\eta_{r}}$ a.s.. Again, by Lemma 2.6, we obtain

$$
\mathbb{E}\left(h\left(X_{\tau_{r^{\prime}}}\right) \mid \mathscr{F}_{\eta_{r}}\right) \leq h\left(X_{\eta_{r}}\right)+(\delta-1) \sum_{\overrightarrow{v u} \in A_{\eta_{r}}} \Delta_{h}(\overrightarrow{v u})-(\delta-1) \mathbb{E}\left(\sum_{\overrightarrow{v u} \in A_{\tau_{r^{\prime}}}} \Delta_{h}(\overrightarrow{v u}) \mid \mathscr{F}_{\eta_{r}}\right) .
$$

Noting that $\mathbb{E}\left(h\left(X_{\tau_{r^{\prime}}}\right) \mid \mathscr{F}_{\eta_{r}}\right) \geq\left[1-\mathbb{P}\left(X_{\tau_{r^{\prime}}} \in F \mid \mathscr{F}_{\eta_{r}}\right)\right] r^{\prime}$, thus we have

$$
\begin{aligned}
\mathbb{P}\left(X_{\tau_{r^{\prime}}} \in F \mid \mathscr{F}_{\eta_{r}}\right) & \geq 1-\frac{h\left(X_{\eta_{r}}\right)}{r^{\prime}}+\frac{\delta-1}{r^{\prime}} \mathbb{E}\left(\sum_{\overrightarrow{v u} \in A_{\tau_{r^{\prime}}} \backslash A_{\eta_{r}}} \Delta_{h}(\overrightarrow{v u}) \mid \mathscr{F}_{\eta_{r}}\right) \\
& \geq 1-\frac{\varepsilon}{2}-(1-\varepsilon)=\frac{\varepsilon}{2} .
\end{aligned}
$$

Therefore, we verify (2.5) by taking $c=\frac{\varepsilon}{2}$ and $\hat{r}=r^{\prime}$ as above, and then finish the proof.

\section{Proof of Theorem 1.1}

Now we apply Theorem 2.1 to prove Theorem 1.1. With each vertex $v$ of $\mathbb{Z} \times\{0,1\}$, we can associate it with coordinates $\mathbf{x}(v) \in \mathbb{Z}, \mathbf{y}(v) \in\{0,1\}$, in the canonical fashion. Set

$$
h(v)=|\mathbf{x}(v)|, F=\{v: \mathbf{x}(v)=0\}, C_{a}=\{(a, i)(a+1, i): i=0,1\}, a \in \mathbb{Z},
$$

where column $C_{a}$ denotes the collection of all horizontal edges connecting all vertices in $\{v: \mathbf{x}(v)=a\}$ with those in $\{v: \mathbf{x}(v)=a+1\}$. One may see $\Delta_{h}(\overrightarrow{v u})=0$ when edge $v u$ is not horizontal, which indicates that we only need to concentrate on edges in column $C_{a}$. Relying on Theorem 2.1, we choose $r^{\prime}$ large enough and separate the left hand side (LHS) of (2.1) into three parts: $\sum_{a=0}^{r-1}, \sum_{a=\tilde{r}}^{r^{\prime}-1}$ and $\sum_{a=r}^{\tilde{r}-1}$, where $r<\tilde{r}<r^{\prime}$ and the exact values of $\tilde{r}$ and $r^{\prime}$ will be specified later. The estimates of the first two parts are similar to those in [8]. We will use a novel technique to show the third part larger than $-\left(1-\varepsilon^{\prime}\right) r^{\prime}$ for some $\varepsilon^{\prime}>0$ based on an observation to be detailed in Lemma 3.4, where the estimate of $\mathbb{E}\left(\sum_{\overrightarrow{v u} \in A_{\tau_{r^{\prime}}} \backslash A_{\eta_{r}}, v u \in C_{a}} \Delta_{h}(\overrightarrow{v u}) \mid \mathscr{F}_{\eta_{r}}\right)$ is transferred to the estimate of $\mathbb{E}\left(\sum_{\overrightarrow{v u} \in A_{\tau_{\infty}} \backslash A_{\eta_{r}}, v u \in C_{a}} \Delta_{h}(\overrightarrow{v u}) \mid \mathscr{F}_{\eta_{r}}\right)$ when $r^{\prime}$ is large enough. The latter is easier to estimate than the former through the iteration technique since the path before $\tau_{\infty}$ has translation invariance property (for details, see Lemma 3.2). Thus, we can determine $\varepsilon^{\prime}$, $\tilde{r}$ and $r^{\prime}$ by the specific estimates in the lemmas below.

Note $\eta_{n}=\inf \left\{t \geq 0: h\left(X_{t}\right)=n\right\}$ for any $n \in \mathbb{N}$, since $h(v)=|\mathbf{x}(v)|$ on $\mathbb{Z} \times\{0,1\}$. Set $\kappa_{n-1}=\inf \left\{t \geq \eta_{n}: h\left(X_{t}\right)=n-1\right\}, n \in \mathbb{N}$ and $\Omega_{n}^{i}=\left\{\mathbf{y}\left(X_{\kappa_{n-1}}\right)=i, \kappa_{n-1}<\infty\right\}$. 
Definition 3.1. Let $\mathbf{X}$ be an ORRW on ladder $\mathbb{Z} \times\{0,1\}$ with reinforcement factor $\delta \in(0,1)$. Then for any $n, n_{0} \in \mathbb{Z}$ and $v_{0} \in V$ satisfying $n>n_{0} \geq 0$ and $h\left(v_{0}\right)=n_{0}$, if $X_{0}=v_{0}$, we define

$$
p^{1}=\mathbb{P}\left(\Omega_{n}^{1-\mathbf{y}\left(X_{\eta_{n}}\right)} \mid \mathscr{F}_{\eta_{n}}\right), p^{0}=\mathbb{P}\left(\Omega_{n}^{\mathbf{y}\left(X_{\eta_{n}}\right)} \mid \mathscr{F}_{\eta_{n}}\right) .
$$

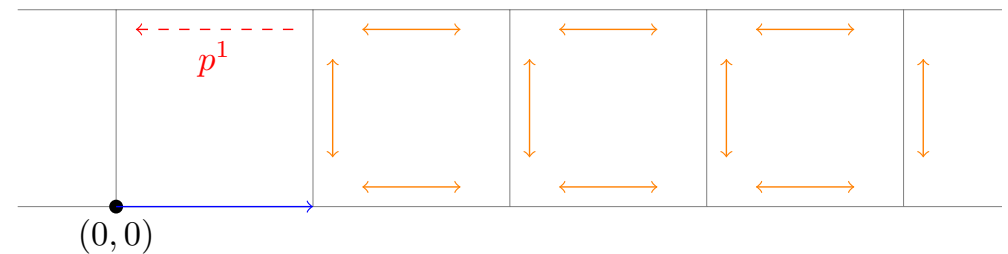

(a)

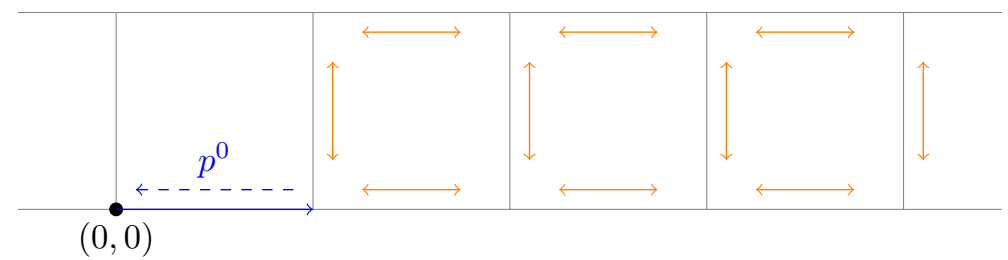

(b)

Figure 1: (a) represents the trajectory of $\mathbf{X}$ in $\Omega_{1}^{1}$, the first step of which is from $(0,0)$ to $(1,0)$ and is indicated by the black real arrow. The red dashed arrow stands for the last step before the hitting time of the board $\{v: h(v)=0\}$. (b) represents the trajectory of $\mathbf{X}$ in $\Omega_{1}^{0}$, the first step of which is from $(0,0)$ to $(1,0)$ and is indicated by the black real arrow. The black dashed arrow is the last step before the hitting time of the board $\{v: h(v)=0\}$.

The conditional probabilities above are well defined since the path from time $\eta_{n}$ to $\kappa_{n-1}$ cannot be influenced by the path before time $\eta_{n}-1$. The performance of the path from time $\eta_{n}$ to $\kappa_{n-1}$ can be seen as an ORRW on half ladder $\mathbb{Z}^{+} \times\{0,1\}$ with the first step from $(0,0)$ to $(0,1)$. What we consider, actually, is the probability the process $\mathbf{X}$ going back to the board $\{v: h(v)=0\}$ from below or above. (See Fig. 1)

Specifically, with $y=0$ or 1 ,

$$
\begin{array}{ll}
\mathbb{P}\left(\Omega_{n}^{1-y} \mid \mathscr{F}_{m}\right)=p^{1} & \text { a.s. on }\left\{\mathbf{y}\left(X_{m}\right)=y, \eta_{n}=m\right\}, \\
\mathbb{P}\left(\Omega_{n}^{y} \mid \mathscr{F}_{m}\right)=p^{0} & \text { a.s. on }\left\{\mathbf{y}\left(X_{m}\right)=y, \eta_{n}=m\right\} .
\end{array}
$$

Here we propose our key lemma whose proof is based on an iterative scheme.

Lemma 3.2. Let $\mathbf{X}$ be an ORRW on ladder $\mathbb{Z} \times\{0,1\}$ with reinforcement factor $\delta \in(0,1)$ and $p^{1}, p^{0}$ be the probabilities given in Definition 3.1. Then

$$
p^{0}-p^{1}<\frac{\delta}{1-\delta}
$$

Proof. Take $n=1, v_{0}=(0,0)$ in Definition 3.1. In fact, $p^{1}=\mathbb{P}\left(\Omega_{1}^{1} \mid X_{1}=(1,0)\right)$ and $p^{0}=\mathbb{P}\left(\Omega_{1}^{0} \mid X_{1}=(1,0)\right)$. Then by the total probability formula, we know that

$$
\begin{aligned}
p^{1}= & \mathbb{P}\left(X_{2}=(2,0) \mid X_{1}=(1,0)\right) \mathbb{P}\left(\Omega_{1}^{1} \mid X_{2}=(2,0), X_{1}=(1,0)\right)+ \\
& \mathbb{P}\left(X_{2}=(1,1) \mid X_{1}=(1,0)\right) \mathbb{P}\left(\Omega_{1}^{1} \mid X_{2}=(1,1), X_{1}=(1,0)\right)+ \\
& \mathbb{P}\left(X_{2}=(0,0) \mid X_{1}=(1,0)\right) \mathbb{P}\left(\Omega_{1}^{1} \mid X_{2}=(0,0), X_{1}=(1,0)\right) \\
= & \frac{1}{2+\delta}\left(P_{\alpha}+P_{\beta}\right),
\end{aligned}
$$


where $P_{\alpha}:=\mathbb{P}\left(\Omega_{1}^{1} \mid X_{2}=(2,0), X_{1}=(1,0)\right), P_{\beta}:=\mathbb{P}\left(\Omega_{1}^{1} \mid X_{2}=(1,1), X_{1}=(1,0)\right)$ and $\mathbb{P}\left(\Omega_{1}^{1} \mid X_{2}=(0,0), X_{1}=(1,0)\right)=0$. Note that $\left\{X_{2}=(2,0), X_{1}=(1,0)\right\} \subset\left\{\mathbf{y}\left(X_{2}\right)=\right.$ $\left.0, \eta_{2}=2\right\}$. By (3.2) we obtain

$$
\begin{aligned}
P_{\alpha} & \geq \mathbb{P}\left(\Omega_{2}^{1} \cap \Omega_{1}^{1} \mid X_{2}=(2,0), X_{1}=(1,0)\right) \\
& =\mathbb{P}\left(\Omega_{2}^{1} \mid X_{2}=(2,0), X_{1}=(1,0)\right) \mathbb{P}\left(\Omega_{1}^{1} \mid X_{2}=(2,0), X_{1}=(1,0), \Omega_{2}^{1}\right) \\
& =p^{1} \cdot \mathbb{P}\left(\Omega_{1}^{1} \mid X_{2}=(2,0), X_{1}=(1,0), X_{\kappa_{1}}=(1,1)\right) \\
& \geq p^{1} \cdot \mathbb{P}\left(X_{\kappa_{1}+1}=(0,1) \mid X_{2}=(2,0), X_{1}=(1,0), X_{\kappa_{1}}=(1,1)\right) \\
& =\frac{1}{2+\delta} p^{1} .
\end{aligned}
$$

Similarly,

$$
\begin{aligned}
P_{\beta} \geq \frac{1}{2+\delta}\left[\mathbb{P}\left(\Omega_{1}^{1} \mid X_{3}=(0,1), X_{2}=(1,1), X_{1}=(1,0)\right)+\right. \\
\left.\mathbb{P}\left(\Omega_{1}^{1} \mid X_{3}=(2,1), X_{2}=(1,1), X_{1}=(1,0)\right)\right] \\
=\frac{1}{2+\delta}\left[1+\mathbb{P}\left(\Omega_{1}^{1} \cap \Omega_{2}^{1} \mid X_{3}=(2,1), X_{2}=(1,1), X_{1}=(1,0)\right)+\right. \\
\left.\mathbb{P}\left(\Omega_{1}^{1} \cap \Omega_{2}^{0} \mid X_{3}=(2,1), X_{2}=(1,1), X_{1}=(1,0)\right)\right] \\
=\frac{1}{2+\delta}\left[1+p^{0} \cdot \mathbb{P}\left(\Omega_{1}^{1} \mid X_{3}=(2,1), X_{2}=(1,1), X_{1}=(1,0), \Omega_{2}^{1}\right)+\right. \\
\left.p^{1} \cdot \mathbb{P}\left(\Omega_{1}^{1} \mid X_{3}=(2,1), X_{2}=(1,1), X_{1}=(1,0), \Omega_{2}^{0}\right)\right],
\end{aligned}
$$

where

$$
\begin{aligned}
\mathbb{P} & \left(\Omega_{1}^{1} \mid X_{3}=(2,1), X_{2}=(1,1), X_{1}=(1,0), \Omega_{2}^{1}\right) \\
& \geq \mathbb{P}\left(X_{\kappa_{1}+1}=(0,1) \mid X_{3}=(2,1), X_{2}=(1,1), X_{1}=(1,0), X_{\kappa_{1}}=(1,1)\right) \\
& =\frac{1}{1+2 \delta}, \\
\mathbb{P} & \left(\Omega_{1}^{1} \mid X_{3}=(2,1), X_{2}=(1,1), X_{1}=(1,0), \Omega_{2}^{0}\right) \\
& \geq \mathbb{P}\left(X_{\kappa_{1}+2}=(0,1), X_{\kappa_{1}+1}=(1,1) \mid X_{3}=(2,1), X_{2}=(1,1), X_{1}=(1,0), X_{\kappa_{1}}=(1,0)\right) \\
& =\frac{1}{1+2 \delta} \cdot \frac{1}{3},
\end{aligned}
$$

which implies $P_{\beta} \geq \frac{1}{2+\delta}\left[1+p^{0} \frac{1}{1+2 \delta}+p^{1} \frac{1}{1+2 \delta} \cdot \frac{1}{3}\right]$. Therefore,

$$
\begin{aligned}
p^{1} & \geq \frac{1}{(2+\delta)^{2}}\left[p^{1}+1+p^{0} \frac{1}{1+2 \delta}+p^{1} \frac{1}{1+2 \delta} \frac{1}{3}\right] \\
& \geq \frac{1}{(2+\delta)^{2}}\left[p^{1}+\left(p^{0}+p^{1}\right)+p^{0} \frac{1}{1+2 \delta}+p^{1} \frac{1}{1+2 \delta} \frac{1}{3}\right],
\end{aligned}
$$

which implies that

$$
p^{1} \geq \frac{2+2 \delta}{\left(\delta^{2}+4 \delta+3\right)(1+2 \delta)+\frac{2}{3}}\left(p^{0}+p^{1}\right) .
$$

Note that $0<\delta<1$ and

$$
\frac{2+2 \delta}{\left(\delta^{2}+4 \delta+3\right)(1+2 \delta)+\frac{2}{3}}-\frac{1-2 \delta}{2(1-\delta)}=\frac{4 \delta^{4}+16 \delta^{3}+7 \delta^{2}-\frac{8}{3} \delta+\frac{1}{3}}{\left[\left(\delta^{2}+4 \delta+3\right)(1+2 \delta)+\frac{2}{3}\right] \cdot 2(1-\delta)},
$$

where $4 \delta^{4}+16 \delta^{3}>0$ and $7 \delta^{2}-\frac{8}{3} \delta+\frac{1}{3}>0$ for any $\delta>0$. This implies $\frac{2+2 \delta}{\left(\delta^{2}+4 \delta+3\right)(1+2 \delta)+\frac{2}{3}}>$ $\frac{1-2 \delta}{2(1-\delta)}$ as $0<\delta<1$. Thus, $p^{1}>\frac{1-2 \delta}{2(1-\delta)}\left(p^{0}+p^{1}\right)$, i.e. as $0<\delta<1$,

$$
p^{1}>(1-2 \delta) p^{0} .
$$


The inequality above implies $p^{0}-p^{1}<2 \delta p^{0}$ and $(2-2 \delta) p^{0}<p^{0}+p^{1}$. Noting that $p^{0}+p^{1} \leq 1$, we obtain that $p^{0}-p^{1}<2 \delta \frac{p^{0}+p^{1}}{2-2 \delta} \leq \frac{\delta}{1-\delta}$ as $0<\delta<1$.

Recall $\eta_{n}=\inf \left\{t \geq 0: h\left(X_{t}\right)=n\right\}$, and let

$$
T_{M, n}=\inf \left\{t \geq \eta_{n}: X_{t} \in\{v: h(v)=n-1 \text { or } n+M\}\right\}, M \in \mathbb{N} .
$$

Definition 3.3. Let $\mathbf{X}$ be an ORRW on ladder $\mathbb{Z} \times\{0,1\}$ with reinforcement factor $\delta \in(0,1)$. Then for any $n, n_{0} \in \mathbb{Z}$ and $v_{0} \in V$ satisfying $n>n_{0} \geq 0$ and $h\left(v_{0}\right)=n_{0}$, if $X_{0}=v_{0}$ we can define

$$
\begin{aligned}
& p_{M}^{1}=\mathbb{P}\left(X_{T_{M, n}}=\left(n-1,1-\mathbf{y}\left(X_{\eta_{n}}\right)\right) \text { or }\left(1-n, 1-\mathbf{y}\left(X_{\eta_{n}}\right)\right) \mid \mathscr{F}_{\eta_{n}}\right), \\
& p_{M}^{0}=\mathbb{P}\left(X_{T_{M, n}}=\left(n-1, \mathbf{y}\left(X_{\eta_{n}}\right)\right) \text { or }\left(1-n, \mathbf{y}\left(X_{\eta_{n}}\right)\right) \mid \mathscr{F}_{\eta_{n}}\right),
\end{aligned}
$$

where $T_{M, n}$ is defined in (3.4).

Note that $p_{M}^{0}$ and $p_{M}^{1}$ are independent of $n$. Moreover,

$$
\begin{aligned}
& \mathbb{P}\left(X_{T_{M, n}}=(n-1,1-y) \mid \mathscr{F}_{1}\right)=p_{M}^{1}, \text { a.s. on }\left\{X_{1}=(n, y), X_{0}=(n-1, y)\right\}, \\
& \mathbb{P}\left(X_{T_{M, n}}=(n-1, y) \mid \mathscr{F}_{1}\right)=p_{M}^{0}, \text { a.s. on }\left\{X_{1}=(n, y), X_{0}=(n-1, y)\right\} .
\end{aligned}
$$

Now we show the asymptotic property for these probabilities. Since

$$
\begin{aligned}
& p_{M}^{1}=\mathbb{P}\left(X_{T_{M, 1}}=(0,1) \mid X_{1}=(1,0), X_{0}=(0,0)\right), \\
& p_{M}^{0}=\mathbb{P}\left(X_{T_{M, 1}}=(0,0) \mid X_{1}=(1,0), X_{0}=(0,0)\right),
\end{aligned}
$$

noting that $X_{T_{M, 1}}(\omega)=(0,1)$ implies $X_{T_{M+1,1}}(\omega)=(0,1)$, we have the following lemma.

Lemma 3.4. Let $\mathbf{X}$ be an ORRW on ladder $\mathbb{Z} \times\{0,1\}$ with reinforcement factor $\delta \in(0,1)$. Let $p_{M}^{1}, p_{M}^{0}$ are probabilities in Definition 3.3. Then as $M \uparrow \infty$,

$$
p_{M}^{1} \uparrow p^{1}, p_{M}^{0} \uparrow p^{0} .
$$

For reader's convenience, we give the following simple property of conditional expectation.

Lemma 3.5. Set $(\Omega, \mathscr{F}, P)$ be a probability space equipped with a filtration $\left(\mathscr{F}_{n}\right)_{n \in \mathbb{N}}$. For any $\left(\mathscr{F}_{n}\right)_{n \in \mathbb{N}}$ stopping times $S_{1}, S_{2}$ and any integrable random variable $f \in \sigma\left(\cup_{n=0}^{\infty} \mathscr{F}_{n}\right)$,

$$
\mathbb{E}\left(f I_{\left\{S_{1}=S_{2}\right\}} \mid \mathscr{F}_{S_{1}}\right)=\mathbb{E}\left(f I_{\left\{S_{1}=S_{2}\right\}} \mid \mathscr{F}_{S_{2}}\right) \text {, a.s. }
$$

At this point, we are ready to conclude the proof of our main theorem.

Proof of Theorem 1.1. $h(v)=|\mathbf{x}(v)|, v \in \mathbb{Z} \times\{0,1\}$ in (3.1) is harmonic (therefore, superharmonic) except on the finite set $F=\{v: h(v)=0\}$. Without loss of generality, let $r$ be a positive integer. Note that $C_{a}$ is given in (3.1), and recall the definition of $\tau_{r^{\prime}}$ in Theorem 2.1.

Firstly, we determine $\varepsilon$ and $r^{\prime}$ on RHS of (2.1). Set

$$
\varepsilon^{\prime}=\frac{1-(1-\delta)\left(p^{0}-p^{1}+1\right)}{2},
$$

then $\varepsilon^{\prime}>0$ by Lemma 3.2. Due to Lemma 3.4, there exists an integer $N>0$ satisfying $\forall n \geq N$,

$$
(\delta-1)\left(p_{n}^{0}-p_{n}^{1}+1\right)>(\delta-1)\left(p^{0}-p^{1}+1\right)-\varepsilon^{\prime} \geq-\left(1-\varepsilon^{\prime}\right) .
$$


Now we choose some $\varepsilon \in\left(0, \varepsilon^{\prime}\right)$. Moreover, for any positive integer $r$, we can take an integer $r^{\prime}>N+r$ such that $(1-\varepsilon) r^{\prime}>\left(1-\varepsilon^{\prime}\right)\left(r^{\prime}-N-r\right)+2(1-\delta)(r+N)$. Set $\tilde{r}=r^{\prime}-N$.

Secondly, we verify that (2.1) holds almost surely on $\left\{\omega: X_{\eta_{r}}(\omega)=(r, 0)\right.$ or $\left.(r, 1)\right\}$. In this situation, $X_{n}$ walks on $\mathbb{Z}^{+} \times\{0,1\}$ during $n \in\left[\eta_{r}, \tau_{r^{\prime}}\right]$. Specifically, the horizontal coordinate of $X_{n}, \mathbf{x}\left(X_{n}\right)=h\left(X_{n}\right)$, and belongs to [0, $\left.r^{\prime}\right]$, if $\eta_{r} \leq n \leq \tau_{r^{\prime}}$.

We now separate $\mathbb{E}\left(\sum_{\overrightarrow{v u} \in A_{\tau_{r^{\prime}}} \backslash A_{\eta_{r}}} \Delta_{h}(\overrightarrow{v u}) \mid \mathscr{F}_{\eta_{r}}\right)$ in (2.1) into three parts according to the subscript $a$ of column $C_{a}: a \in[0, r-1], a \in\left[\tilde{r}, r^{\prime}-1\right]$ and $a \in[r, \tilde{r}-1]$ (see Fig. 2), and estimate them respectively.

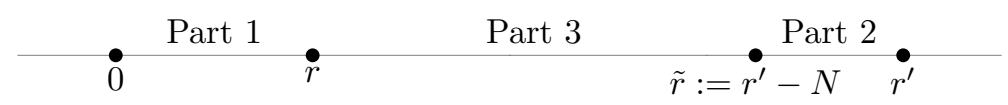

Figure 2: An intuitive illustration of the three parts separated in LHS of (2.1) and the notation of $\tilde{r}$.

Part 1 and Part 2: Noting that each column $C_{a}$ has only two horizontal edges and for any integer $a \in[0, r-1] \cup\left[\tilde{r}, r^{\prime}-1\right]$, we have

$$
(\delta-1) \mathbb{E}\left(\sum_{\overrightarrow{v u} \in A_{\tau_{r^{\prime}}} \backslash A_{\eta_{r}}, v u \in C_{a}} \Delta_{h}(\overrightarrow{v u}) \mid \mathscr{F}_{\eta_{r}}\right) \geq 2(\delta-1) .
$$

Part 3: For any integer $a \in[r, \tilde{r}-1], r-a-1 \geq N$, thus $\tau_{a+1} \leq \tau_{r^{\prime}}$. Noting that $h\left(X_{t}\right)<a$ for any $t \leq \eta_{r}$, we have $\eta_{a+1}=\inf \left\{t>\eta_{r}: h\left(X_{t}\right)=a+1\right\}$, which implies $\eta_{r}<\tau_{a+1} \leq \eta_{a+1}$.

Let

$$
D_{a}^{k}=\left\{\omega: \sum_{\overrightarrow{v u} \in A_{\tau_{r^{\prime}}} \backslash A_{\eta_{r}}, v u \in C_{a}} \Delta_{h}(\overrightarrow{v u})=k\right\}, k=0,1,2 .
$$

One can see that $\cup_{k=0}^{2} D_{a}^{k}=\Omega$ and $D_{a}^{i} \cap D_{a}^{j}=\emptyset$ for $i \neq j . \omega \in D_{a}^{1} \cup D_{a}^{2}$ indicates that there is an edge in $C_{a}$ traversed by $X_{n}(\omega)$ between stopping time $\eta_{r}$ and $\tau_{r^{\prime}}$, thus $D_{a}^{1} \cup D_{a}^{2} \subseteq\left\{h\left(X_{\tau_{a+1}}\right)=a+1\right\}=\left\{\tau_{a+1}=\eta_{a+1}\right\}$. Then Lemma 3.5 implies that

$$
\begin{aligned}
& (\delta-1) \mathbb{E}\left(\sum_{\overrightarrow{v u} \in A_{\tau_{r^{\prime}}} \backslash A_{\eta_{r}}, v u \in C_{a}} \Delta_{h}(\overrightarrow{v u}) \mid \mathscr{F}_{\tau_{a+1}}\right) \\
& =(\delta-1)\left[2 \mathbb{P}\left(D_{a}^{2} \mid \mathscr{F}_{\tau_{a+1}}\right)+\mathbb{P}\left(D_{a}^{1} \mid \mathscr{F}_{\tau_{a+1}}\right)+0 \cdot \mathbb{P}\left(D_{a}^{0} \mid \mathscr{F}_{\tau_{a+1}}\right)\right] \\
& =(\delta-1)\left[2 \mathbb{P}\left(D_{a}^{2}, \tau_{a+1}=\eta_{a+1} \mid \mathscr{F}_{\tau_{a+1}}\right)+\mathbb{P}\left(D_{a}^{1}, \tau_{a+1}=\eta_{a+1} \mid \mathscr{F}_{\tau_{a+1}}\right)\right] \\
& \stackrel{a . s .}{=}(\delta-1)\left[2 \mathbb{P}\left(D_{a}^{2}, \tau_{a+1}=\eta_{a+1} \mid \mathscr{F}_{\eta_{a+1}}\right)+\mathbb{P}\left(D_{a}^{1}, \tau_{a+1}=\eta_{a+1} \mid \mathscr{F}_{\eta_{a+1}}\right)\right] .
\end{aligned}
$$

Note that the fact $T_{r^{\prime}-a-1, a+1}=\inf \left\{t \geq \eta_{a+1}: h\left(X_{t}\right)=a\right.$ or $\left.r^{\prime}\right\}$ implies $\left\{D_{a}^{2}, \tau_{a+1}=\right.$ $\left.\eta_{a+1}\right\} \subset\left\{X_{T_{r^{\prime}-a-1, a+1}}=\left(a, \mathbf{y}\left(X_{\eta_{a+1}}\right)\right), \tau_{a+1}=\eta_{a+1}\right\}$. Thus

$$
\begin{aligned}
& \mathbb{P}\left(D_{a}^{2}, \tau_{a+1}=\eta_{a+1} \mid \mathscr{F}_{\eta_{a+1}}\right) \\
& =\mathbb{P}\left(D_{a}^{2}, X_{T_{r^{\prime}-a-1, a+1}}=\left(a, \mathbf{y}\left(X_{\eta_{a+1}}\right)\right), \tau_{a+1}=\eta_{a+1} \mid \mathscr{F}_{\eta_{a+1}}\right) .
\end{aligned}
$$

Meanwhile, $\left\{X_{T_{r^{\prime}-a-1, a+1}}=\left(a, \mathbf{y}\left(X_{\eta_{a+1}}\right)\right), \tau_{a+1}=\eta_{a+1}\right\},\left\{h\left(X_{T_{r^{\prime}-a-1, a+1}}\right)=r^{\prime}, \tau_{a+1}=\right.$ $\left.\eta_{a+1}\right\}$ and $\left\{X_{T_{r^{\prime}-a-1, a+1}}=\left(a, 1-\mathbf{y}\left(X_{\eta_{a+1}}\right)\right), \tau_{a+1}=\eta_{a+1}\right\}$ are disjoint sets, and their union 
is $\left\{\tau_{a+1}=\eta_{a+1}, h\left(X_{\eta_{r}}\right)=r\right\} \supset D_{a}^{1}$. Observing $\left\{X_{T_{r^{\prime}-a-1, a+1}}=\left(a, 1-\mathbf{y}\left(X_{\eta_{a+1}}\right)\right), \tau_{a+1}=\right.$ $\left.\eta_{a+1}\right\} \subset D_{a}^{0}$ and $D_{a}^{0} \cap D_{a}^{1}=\emptyset$, we obtain that

$$
\begin{array}{rl}
\mathbb{P}\left(D_{a}^{1}, \tau_{a+1}=\eta_{a+1} \mid \mathscr{F}_{\eta_{a+1}}\right)= & \mathbb{P}\left(D_{a}^{1}, X_{T_{r^{\prime}-a-1, a+1}}=\left(a, \mathbf{y}\left(X_{\eta_{a+1}}\right)\right), \tau_{a+1}=\eta_{a+1} \mid \mathscr{F}_{\eta_{a+1}}\right) \\
& +\mathbb{P}\left(D_{a}^{1}, h\left(X_{T_{r^{\prime}-a-1, a+1}}\right)=r^{\prime}, \tau_{a+1}=\eta_{a+1} \mid \mathscr{F}_{\eta_{a+1}}\right) \\
\leq \quad 2 & \mathbb{P}\left(D_{a}^{1}, X_{T_{r^{\prime}-a-1, a+1}}=\left(a, \mathbf{y}\left(X_{\eta_{a+1}}\right)\right), \tau_{a+1}=\eta_{a+1} \mid \mathscr{F}_{\eta_{a+1}}\right) \\
& +\mathbb{P}\left(h\left(X_{T_{r^{\prime}-a-1, a+1}}\right)=r^{\prime}, \tau_{a+1}=\eta_{a+1} \mid \mathscr{F}_{\eta_{a+1}}\right) .
\end{array}
$$

Combining (3.10), (3.11) and $\mathbb{P}\left(D_{a}^{0}, X_{T_{r^{\prime}-a-1, a+1}}=\left(a, \mathbf{y}\left(X_{\eta_{a+1}}\right)\right), \tau_{a+1}=\eta_{a+1} \mid \mathscr{F}_{\eta_{a+1}}\right) \geq$ 0 , and noting $\delta-1<0$, we have that

$$
\begin{aligned}
& \geq \quad(\delta-1)\left[2 \sum_{k=0}^{2} \mathbb{P}\left(D_{a}^{k}, X_{T_{r^{\prime}-a-1, a+1}}=\left(a, \mathbf{y}\left(X_{\eta_{a+1}}\right)\right), \tau_{a+1}=\eta_{a+1} \mid \mathscr{F}_{\eta_{a+1}}\right)\right. \\
& \left.+\mathbb{P}\left(h\left(X_{T_{r^{\prime}-a-1, a+1}}\right)=r^{\prime}, \tau_{a+1}=\eta_{a+1} \mid \mathscr{F}_{\eta_{a+1}}\right)\right] \\
& =\quad(\delta-1)\left[2 \mathbb{P}\left(X_{T_{r^{\prime}-a-1, a+1}}=\left(a, \mathbf{y}\left(X_{\eta_{a+1}}\right)\right), \tau_{a+1}=\eta_{a+1} \mid \mathscr{F}_{\eta_{a+1}}\right)\right. \\
& \left.\quad+\mathbb{P}\left(h\left(X_{T_{r^{\prime}-a-1, a+1}}\right)=r^{\prime}, \tau_{a+1}=\eta_{a+1} \mid \mathscr{F}_{\eta_{a+1}}\right)\right] \\
& \stackrel{a . s .}{=} \quad(\delta-1)\left[2 p_{r^{\prime}-a-1}^{0}+\left(1-p_{r^{\prime}-a-1}^{0}-p_{r^{\prime}-a-1}^{1}\right)\right] \cdot I_{\left\{\tau_{a+1}=\eta_{a+1}\right\}} I_{\left\{\eta_{a+1}<\infty\right\}}
\end{aligned}
$$

(by $(3.7))>-\left(1-\varepsilon^{\prime}\right)$.

Therefore, summing up these three parts, we get that on $\left\{\omega: X_{\eta_{r}}(\omega)=(r, 0)\right.$ or $\left.(r, 1)\right\}$,

$$
\begin{aligned}
& (\delta-1) \mathbb{E}\left(\sum_{\overrightarrow{v u} \in A_{\tau_{r^{\prime}}} \backslash A_{\eta_{r}}} \Delta_{h}(\overrightarrow{v u}) \mid \mathscr{F}_{\eta_{r}}\right) \\
& =\left(\sum_{a=0}^{r-1}+\sum_{a=r^{\prime}-N}^{r^{\prime}-1}\right)(\delta-1) \mathbb{E}\left(\sum_{\overrightarrow{v u} \in A_{\tau_{r^{\prime}}} \backslash A_{\eta_{r}}, v u \in C_{a}} \Delta_{h}(\overrightarrow{v u}) \mid \mathscr{F}_{\eta_{r}}\right) \\
& \quad+\sum_{a=r}^{r^{\prime}-N-1}(\delta-1) \mathbb{E}\left[\mathbb{E}\left(\sum_{\overrightarrow{v u} \in A_{\tau_{r^{\prime}}} \backslash A_{\eta_{r}}, v u \in C_{a}} \Delta_{h}(\overrightarrow{v u}) \mid \mathscr{F}_{\tau_{a+1}}\right) \mid \mathscr{F}_{\eta_{r}}\right] \\
& \left.\left.\begin{array}{l}
a . s . \\
\geq
\end{array}\right]-\left(1-\varepsilon^{\prime}\right)\left(r^{\prime}-N-r\right)-2(1-\delta)(r+N)\right] \\
& \geq-(1-\varepsilon) r^{\prime} .
\end{aligned}
$$

Finally, we can also verify (3.12) on $\left\{\omega: X_{\eta_{r}}(\omega)=(-r, 0)\right.$ or $\left.(-r, 1)\right\}$ by the same approach to work on $\mathbb{Z}^{-} \times\{0,1\}$ and finish proving Theorem 1.1 by Theorem 2.1.

\section{Conclusion}

Given any $n \in \mathbb{N}$, it is expected that $\delta$-ORRW on ladder $\mathbb{Z} \times\{0, \ldots, n\}$ with $n+1$ levels is almost surely recurrent for any $\delta>0$ ([7]). Theorem 1.1 together with the results in [7] and [8] confirm this is true for $n=1$.

For $n \geq 2$, our method seems to have met obstacles. In this case, we can still apply Theorem 2.1 and separate LHS of (2.1) into three parts: $\sum_{a=0}^{r-1}, \sum_{a=\tilde{r}}^{r^{\prime}-1}$ and $\sum_{a=r}^{\tilde{r}-1}$, where 
$r<\tilde{r}<r^{\prime}$. The estimation approach of the first two parts is still applicable. While for the third part, our proposed approach is to decompose the paths into $D_{a}^{k}, k=0,1, \ldots, n+1$ according to the numbers of the horizontal edges of column $C_{a}$ traversed firstly from left to right, and then to compute probabilities related to each $D_{a}^{k}$. At present, we are only able to get the following type of weaker results: there are $\delta_{1}, \delta_{2}$ depending on $n$ such that $0<\delta_{1}<\frac{n}{n+1}<\frac{n}{n-1}<\delta_{2}$, and $\delta$-ORRW is a.s. recurrent for any $\delta \in\left(\delta_{1}, \delta_{2}\right)$. Such a result was already claimed in [8]. Fortunately, for $\mathbb{Z} \times\{0,1\}$, Lemma 3.2 holds and provides some accurate estimates of probabilities related to each $D_{a}^{k}$ for any $\delta \in(0,1)$, and thus we can prove Theorem 1.1.

To handle the case $n \geq 2$, new tools need to be developed, for example, decomposing paths more delicately in a larger column (e.g. $(n+1) \times(n+1)$ square) to get inequality similar to Lemma 3.2.

Moreover, if we assume that ORRWs on $\mathbb{Z} \times\{-n, \ldots,-1,0,1, \ldots, n\}$ are a.s. recurrent for any $n \in \mathbb{N}$, an interesting problem will arise naturally: Can one deduce a.s. recurrence for ORRWs on $\mathbb{Z}^{2}$ ?

There are plenty of differences between reinforced random walks and Markov Chains. For instance, unlike Markov Chains, whether or not the transience/recurrence 0-1 law of reinforced random walks holds is a subtle problem. In particular, although the known results of ORRWs show the transience/recurrence $0-1$ law, there seems to be lack of deep insights on this property of general cases, and it is unknown that $\delta$-ORRW on $\mathbb{Z}^{d}$ with $d \geq 2$ satisfies the transience/recurrence 0 - 1 law for all $\delta>0$.

\section{References}

[1] Coppersmith, D. and Diaconis, P.: Random walk with reinforcement. Unpublished, (1987).

[2] Collevecchio, A., Kious, D. and Sidoravicius, V.: The branching-ruin number and the critical parameter of once-reinforced random walk on trees. Comm. Pure Appl. Math. 73(1), (2020), 210-236. MR-4033893

[3] Davis, B.: Reinforced random walk. Probab. Theory Related Fields. 84(2), (1990), 203-229. MR-1030727

[4] Durrett, R.: Probability: Theory and Examples (4th edition). Cambridge University Press, Cambridge, 2010. $\mathrm{x}+428$ pp. MR-2722836

[5] Kious, D., Schapira, B. and Singh, A.: Once reinforced random walk on $\mathbb{Z} \times \Gamma$. arXiv:1807.07167v1 (2018).

[6] Kious, D. and Sidoravicius, V.: Phase transition for the once-reinforced random walk on $\mathbb{Z}^{d}$-like trees. Ann. Probab. 46(4), (2018), 2121-2133. MR-3813987

[7] Sellke, T.: Recurrence of reinforced random walk on a ladder. Electron. J. Probab. 11, (2006), 301-310. MR-2217818

[8] Vervoort, M. R.: Reinforced random walks. Unpublished, (2002), https://staff.fnwi.uva.nl/m.r. vervoort/walk.pdf.

Acknowledgments. We thank the anonymous referees and the editor for their comments which improved greatly the quality of the paper, and thank Prof. Pierre Tarrès, Prof. Yuan Zhang for their suggestions and Dr. Yuelin Liu for her helps. 